\title{
THE IDENTIFICATION OF A TIME DEPENDENT SORPTION PARAMETER FROM SOIL COLUMN EXPERIMENTS*
}

\author{
K. RENEE FISTER ${ }^{\dagger}$, MAEVE L. MCCARTHY ${ }^{\dagger}$, AND SETH F. OPPENHEIMER ${ }^{\ddagger}$
}

\begin{abstract}
Soil column studies are used frequently in seeking to understand the behavior of a particular contaminant in a saturated homogeneous soil of a given type. The concentration of the contaminant is modeled by a parabolic partial differential equation. We seek to identify the sorption partitioning coefficient as a function of time from limited boundary data. We discuss an output least squares formulation of the problem with Tikhonov regularization. We explicitly characterize a source condition that determines the rate of convergence of the method. Numerical examples are presented.
\end{abstract}

Key words. parameter identification, inverse problems, Tikhonov regularization, parabolic partial differential equation, sorption partitioning

AMS subject classifications. 35K57, 35R30, 47A52

DOI. $10.1137 / 050626302$

1. Introduction. The purpose of this paper is to develop theoretical and numerical approaches for approximating an unknown time dependent parameter in a parabolic partial differential equation, given limited boundary data. The equation we shall be working with is a model of a soil column study. These column studies are used frequently in seeking to understand the behavior of a particular contaminant in a saturated homogeneous soil of a given type. The parameter we are seeking to approximate is the sorption partitioning coefficient. This parameter is a measure of the proportion of contaminant that is bound to the soil. In isothermal situations when there are no other contaminants present, the partitioning coefficient is usually taken to be constant. However, if there is another contaminant, e.g., sea salt, or if the temperature is changing, the partitioning coefficient may change as well. Therefore, strictly speaking, the partitioning coefficient is a function of some physical factor other than time. However, if we understand the controlling physical factor as a function of time, we may treat the partitioning coefficient also as a function of time and deduce the true physical functional relationship after the partitioning coefficient has been found as a function of time.

This approach will allow us considerable savings in time and resources when determining how a partitioning coefficient varies with different physical factors. These savings will result from identifying the partitioning coefficient's dependence on the relevant physical factor by means of a single column experiment rather then a large number of separate batch tests.

Section 2 shall be devoted to a discussion of the model, the simplifying assumptions that are applicable, and various necessary facts about the forward problem.

\footnotetext{
*Received by the editors March 8, 2005; accepted for publication (in revised form) December 28, 2005; published electronically May 3, 2006.

http://www.siam.org/journals/siap/66-4/62630.html

${ }^{\dagger}$ Department of Mathematics and Statistics, Murray State University, 6C Faculty Hall, Murray, KY 42071 (renee.fister@murraystate.edu, maeve.mccarthy@murraystate.edu). The first author was supported in part by National Science Foundation grant DMS 0414011. The second author was supported in part by National Science Foundation grant DMS 0209562.

${ }^{\ddagger}$ Department of Mathematics and Statistics, Mississippi State University, P. O. Drawer MA, Mississippi State, MS 39762 (seth@math.msstate.edu). This author was supported in part by National Institutes of Health grant DHHS/NIH P20 RR17661. This is Center for Environmental Health Sciences Publication 109.
} 
Section 3 establishes identifiability of the sorption partitioning coefficient from the available experimental data and applies an output least squares method with Tikhonov regularization to the parameter identification problem. The method is tested in section 4 .

2. Discussion of the model. The general approach to modeling convectiondiffusion-sorption models may be found in the work of Leij and Dane [12] and Domenico and Schwartz [6]. Sorption of mixtures was treated by Oppenheimer [15]. A thorough treatment of the modeling of the sorption process may be found in Oppenheimer, Kingery, and Han [16]. Column studies are discussed in detail by Adrian, Ozkan, and Alshawabkeh [1].

We model the one dimensional flow of water with a dissolved contaminant through a soil column. The contaminant can be dissolved in the water or bound, that is sorbed, to the soil. We will assume cylindrical symmetry in the column to reduce the problem to one spatial dimension. The column will be assumed to have length $L$ and is modeled as the interval $[0, L]$. The distance from the inflow end of the column will be given by $z$. The time since the start of the experiment will be given by $\tau$. At spatial point $z$ and time $\tau$, the solution concentration (in mass of contaminant per unit volume of water) is denoted $c(z, \tau)$, and the sorbed concentration (in mass of contaminant per unit mass of soil) is denoted $q(z, \tau)$.

We will assume the equilibrium relationship $q=f(c)$. The function $f$ is called the equilibrium isotherm.

We will assume the following physical values: $\rho_{V}(z)$ is the void density per unit volume at spatial coordinate $z, \rho_{S}(z)$ is the soil mass density per unit volume, $A$ is the cross-sectional area of the column, $v$ is the fluid velocity, and $D$ is the diffusivity constant that appears in Fick's law for diffusion. Fick's law assumes that the rate of diffusion of the contaminant in the fluid is given by $-D \partial c / \partial z$.

Choosing $z$ to be any interior point of the column, $\tau$ any positive time, and $\Delta z$ and $\Delta \tau$ small positive numbers, the change in the amount of contaminant stored in the section of the column $[z, z+\Delta z]$ between $\tau$ and $\tau+\Delta \tau$ is given by

$$
\begin{aligned}
\int_{z}^{z+\Delta z} & {\left[A \rho_{V}(\eta) c(\eta, \tau+\Delta \tau)+A \rho_{S}(\eta) q(\eta, \tau+\Delta t)\right.} \\
& \left.-\left(A \rho_{V}(\eta) c(\eta, \tau)+A \rho_{S}(\eta) q(\eta, \tau)\right)\right] d \eta
\end{aligned}
$$

This will equal the total inward flux less the outward flux at $z$ and $z+\Delta z$ over the time from $\tau$ to $\tau+\Delta \tau$,

$$
\begin{aligned}
\int_{\tau}^{\tau+\Delta \tau}[ & v A \rho_{V}(z) c(z, s)-D A \rho_{V}(z) \frac{\partial c}{\partial z}(z, s) \\
& \left.-\left(v A \rho_{V}(z+\Delta z) c(z+\Delta z, s)-D A \rho_{V}(z+\Delta z) \frac{\partial c}{\partial z}(z+\Delta z, s)\right)\right] d s
\end{aligned}
$$

Setting the two expressions equal, dividing by $\Delta z \Delta \tau$, and letting $\Delta z$ and $\Delta \tau$ tend to zero yields

$$
A\left[\rho_{V}(z) c(z, \tau)+\rho_{S}(z) q(z, \tau)\right]_{\tau}=-v A\left[\rho_{V}(z) c(z, \tau)\right]_{z}+D A\left[\rho_{V}(z) c_{z}(z, \tau)\right]_{z} .
$$

If we assume that the mass density $\rho_{S}=M /(L A)$ and the pore volume density $\rho_{V}=V /(L A)$ are constant, where $M$ is the total mass of soil in the cylinder and $V$ 
is the total void space in the cylinder, we obtain

$$
[V c+M q]_{\tau}=-v V c_{z}+D V c_{z z} .
$$

We have already described the expected equilibrium relationship between the sorbed concentration, $q$, and the solution concentration, which is given by $q=f(c)$. Since the solution concentration is changing in time we must either assume that this equilibrium relationship holds even as $c$ changes in time or we must specify how $q$ changes as $c$ changes. For completeness we will describe the nonequilibrium modeling approach before we make our final assumptions. The standard model [16, 12] for sorption when $c$ is known at each time $t$ is

$$
\frac{\partial q}{\partial \tau}=r F(c, q),
$$

where $r$ is a sorption rate constant and $F$ satisfies the following requirements: If $q<f(c)$, then $F$ is positive; if $q=f(c)$, then $F=0$; and if $q>f(c)$, then $F$ is negative. Some typical examples of the isotherm $f$ are the Henry or linear isotherm

$$
f(c)=\xi c,
$$

the Langmuir isotherm

$$
f(c)=\frac{\xi c}{1+\beta c},
$$

and the Freundlich isotherm

$$
f(c)=\xi c^{\gamma} .
$$

An example of $F$ is a simple reversible sink

$$
F(c, q)=f(c)-q,
$$

where the rates of sorption and desorption are the same and where, regardless of whether the process is sorbing or disorbing, the same fixed $c$ value will yield the same equilibrium point. Another example of $F$ is a simple irreversible sink

$$
(f(c)-q)^{+},
$$

where there is hysteresis occurring, and while a contaminant can be sorbed, it cannot be desorbed. There are a wide variety of such models, and the reader is referred to [16].

When the local kinetics (2.2) are combined with the conservation-of-mass equation (2.1) previously derived, we obtain

$$
\begin{aligned}
V \frac{\partial c}{\partial \tau}+M \frac{\partial q}{\partial \tau} & =-v V \frac{\partial c}{\partial z}+D V \frac{\partial^{2} c}{\partial z^{2}}, \\
\frac{\partial q}{\partial \tau} & =r F(c, q) .
\end{aligned}
$$

The equilibrium partitioning assumption is that $r$ is much larger than $D$ and $v$. Dividing the second system by $r$ and defining $\varepsilon=1 / r$, we may consider this a singular perturbation problem:

$$
\begin{aligned}
V \frac{\partial c}{\partial \tau}+M \frac{\partial q}{\partial \tau} & =-v V \frac{\partial c}{\partial z}+D V \frac{\partial^{2} c}{\partial z^{2}} \\
\varepsilon \frac{\partial q}{\partial \tau} & =F(c, q) .
\end{aligned}
$$


Since we will consider only cases where we are close to equilibrium, we need consider only the outer solution to the unperturbed problem

$$
F(c, q)=0 \quad \text { or } \quad q=f(c) .
$$

Thus, we can replace system (2.4) with

$$
\frac{\partial}{\partial \tau}(V c+M f(c))=-v V \frac{\partial c}{\partial z}+D V \frac{\partial^{2} c}{\partial z^{2}} .
$$

In this paper we will accept the equilibrium partitioning assumption and use a Henry isotherm. The linear partitioning assumption is usually valid when concentrations are low. The common set of boundary conditions that we will be using is

$$
c(0, \tau)=0, \quad \frac{\partial c}{\partial z}(L, \tau)=0 .
$$

The first boundary condition is used to model the case where the inflow of water contains no contaminant, and the second boundary condition models the fact that there is no diffusion across the end of the column, only convection.

We will perform the standard change of variables [12] with respect to time and length by introducing new variables

$$
t=v \tau / L \quad \text { and } \quad x=z / L .
$$

Using these new variables, we obtain the form of the model we wish to study:

$$
\begin{aligned}
\frac{\partial}{\partial t}(\beta c) & =-\frac{\partial c}{\partial x}+K \frac{\partial^{2} c}{\partial x^{2}}, \\
c(0, t) & =0, \\
\frac{\partial c}{\partial x}(1, t) & =0, \\
c(x, 0) & =c_{0}(x),
\end{aligned}
$$

where

$$
\beta=1+\frac{\xi M}{V}
$$

and

$$
K=\frac{D}{v L}
$$

is the nondimensionalized diffusion coefficient. The pseudotime variable measures pore volumes; that is, $t=1$ is the time it takes the flow to move from the top of the column to the bottom of the column.

In column studies, measurements of the exit solution concentration are taken. Therefore, the extra information available is a sequence of $N$ time measurements taken at the end of the column $x=1$,

$$
c\left(1, t_{1}\right), \ldots, c\left(1, t_{N}\right) .
$$

It is worthwhile to briefly discuss how the forward model came to be. The same model, with constant $\beta$, was successfully used to model column studies of fresh water 
sediments. However, the constant $\beta$ model failed when dealing with salt water sediments, and it was hypothesized by Myers [13] that $\beta$ was changing with the saline concentration. Since it was expected that the saline concentration would equalize much more quickly than the concentration of the contaminant being studied, we chose to approximate the salt concentration as being spatially uniform and, thus, $\beta$ as spatially uniform. In the technical report on this approach [14], we used a decreasing exponential ansatz for the saline concentration, assuming that the concentration would be dominated by the decay in the first eigenfunction. This yielded model fits that were considered reasonable by the engineers on the project. Henceforth, we assume that $\xi$, and hence $\beta$, is changing with time.

The initial contaminant concentration is taken to be spatially constant, $c(x, 0)=$ $c_{0}>0$, because the samples have time to equilibrate before the experiment begins. It is worth noting that this initial contaminant concentration, while physically accurate, does not meet the boundary condition. Indeed, in the explicit solutions generated for the technical report [14], there is a Gibb's phenomenon. Fortunately the problem is governed by a parabolic evolution operator, and solutions satisfy the boundary conditions for all positive times.

2.1. Contaminant mass constraint. We will now compute the value $\beta(0)$, which is related to the equilibrium coefficient $\xi$; see (2.6). We will assume that we know the total mass of soil in the column $M$, the total volume of water in the column $V$, the cross-sectional area of the column $A$, the diffusivity constant $D$, and the fluid velocity $v$. We will also assume that we know that the initial solution concentration is a constant, $c_{0}$. The mass flow of contaminant out of the tube at time $t$ will be given by

$$
A c(1, t) V / A \text {. }
$$

Thus, if we let the process continue until almost all of the contaminant has been flushed from the column at time $t_{N}$, we have that the total mass of contaminant present in the column at time $t=0$ will be approximately

$$
\int_{0}^{t_{N}} c(1, t) V d t .
$$

We also know that the total mass of contaminant at time $t=0$ will be given by

$$
\beta(0) c_{0} V .
$$

Equating the expressions in (2.7) and (2.8), we obtain

$$
\beta(0) \approx \frac{1}{c_{0}} \int_{0}^{t_{N}} c(1, t) d t .
$$

We need to add a caveat at this point. The physical system allows contaminant to leave the cylinder only through the boundary at $x=1$. However, examining the original system and integrating with respect to $x$ yields

$$
\begin{aligned}
\frac{\partial}{\partial t} \int_{0}^{1}(\beta c) d x & =c(0, t)-c(1, t)+K \frac{\partial c}{\partial x}(1, t)-K \frac{\partial c}{\partial x}(0, t) \\
& =-c(1, t)-K \frac{\partial c}{\partial x}(0, t)
\end{aligned}
$$


Integrating the previous equation with respect to $t$ from 0 to $t_{N}$ and assuming that the $c\left(x, t_{N}\right) \approx 0$ yields

$$
\beta(0) c_{0} \approx \int_{0}^{t_{N}} c(1, t) d t+\int_{0}^{t_{N}} K \frac{\partial c}{\partial x}(0, t) d t
$$

Finally, solving for $\beta(0)$, we have

$$
\beta(0)=\frac{1}{c_{0}} \int_{0}^{t_{N}} c(1, t) d t+\frac{1}{c_{0}} \int_{0}^{t_{N}} K \frac{\partial c}{\partial x}(0, t) d t .
$$

Thus, the model allows for contaminant to leave the cylinder at $x=0$, which will give some discrepancy. We require that $D \ll v$, which implies that $K \approx 0$, in order to minimize this error.

3. Identification of the sorption coefficient. The forward problem is

$$
\begin{aligned}
(\beta c)_{t} & =-c_{x}+K c_{x x}, & 0<x<1,0<t<T=t_{N}, \\
c(0, t) & =0, \quad c_{x}(1, t)=0, & 0<t<T, \\
c(x, 0) & =c_{0}(x), & 0<x<1 .
\end{aligned}
$$

Our goal is to estimate the parameter $\beta$ from noisy measurements of $c(1, t)$. Although identification problems for parabolic equations have been addressed both theoretically $[11,2,3,5,18]$ and numerically $[4,10]$, the general framework is to consider the problem

$$
\begin{array}{rlrl}
u_{t} & =L(a)[u] & \text { in } \Omega \times[0, T], \\
u(x, 0) & =u_{0}(x) & & \text { on } \Omega, \\
G(a)[u] & =0 & & \text { on } \partial \Omega \times[0, T],
\end{array}
$$

subject to additional information $B[u]=0$ on $\Omega \times[0, T]$ or $\partial \Omega \times[0, T]$. The spatial operators $L, G, B$ may be linear or quasi-linear. The unknown coefficient $a$ may be part of $L$ or $G$ and may depend on $x, t$, or $u$. The goal is to recover $a$ from information about $u$, the solution of (3.2). Although our forward problem (3.1) can be transformed into the form (3.2) by setting $u=\beta c$, the boundary data available from our experiment is $c(1, t)$. Thus boundary data for the transformed problem $u(1, t)$ would require knowledge of $\beta$, the parameter we seek. Similar issues arise with other transformation approaches. As in [3], trace-type functionals can then be used to establish existence of a solution. This approach can also be implemented numerically [10]. However, the dependence of the operators on nonlocal information can lead to numerical instabilities. We wish to develop an algorithm that uses our available data directly and avoids the use of nonlocal information. We begin by establishing identifiability of the parameter $\beta$ from the available data $c(1, t)$. We apply output least squares with Tikhonov regularization to this problem. We investigate the rate of convergence and determine an appropriate source condition.

3.1. Identifiability. Recall the contaminant mass constraint (2.9),

$$
\beta(0) \approx \frac{1}{c_{0}} \int_{0}^{t_{N}} c(1, t) d t
$$

discussed in section 2.1. As a consequence of this and the fact that our data is $c(1, t)$, it is reasonable to assume that $\beta(0)$ is fixed and to let

$$
\mathcal{B}=\left\{\beta \in H^{1}(0, T) \mid 0<m<\beta(t)<\mathcal{M}, \beta(0)=b\right\} .
$$


Our existence result follows from the application of standard results; see [20, 9].

Theorem 3.1. If $\beta \in \mathcal{B}$, then $c_{\beta} \in W=L^{2}\left((0, T) ; H^{1}(0,1)\right)$ and $c_{\beta}(1, \cdot) \in$ $L^{2}(0, T)$.

In order to establish the identifiability of $\beta$ we must establish the injectivity of the parameter-to-output map

$$
\beta \rightarrow \gamma c_{\beta},
$$

where $\gamma$ denotes the trace operator

$$
\gamma: L^{2}\left((0, T) ; H^{1}(0,1)\right) \rightarrow L^{2}(0, T), \quad \gamma c=c(1, t) .
$$

TheOREM 3.2. Let $c_{1}(x, t)$ and $c_{2}(x, t) \in W$ be solutions of the direct problem (3.1) corresponding to $\beta_{1}(t)$ and $\beta_{2}(t) \in \mathcal{B}$. If $\gamma c_{1}=\gamma c_{2}$, then $\beta_{1}(t)=\beta_{2}(t)$ for all $t \in[0, T]$.

Proof. Use $\beta_{1}, c_{1}$ and $\beta_{2}, c_{2}$ in (3.1) and subtract to find

$$
\left(\beta_{1} c_{1}-\beta_{2} c_{2}\right)_{t}=-\left(c_{1}-c_{2}\right)_{x}+K\left(c_{1}-c_{2}\right)_{x x} .
$$

Let $\phi=\beta_{1} c_{1}-\beta_{2} c_{2}$ and rearrange terms

$$
\phi_{t}=-\frac{1}{\beta_{1}} \phi_{x}+\frac{K}{\beta_{1}} \phi_{x x}+\frac{\left(\beta_{2}-\beta_{1}\right)}{\beta_{1}}\left(\mathcal{A} c_{2}\right),
$$

where $\mathcal{A} c=-c_{x}+K c_{x x}$. Multiply by $\phi$ and integrate with respect to $x$ :

$$
\begin{aligned}
\int_{0}^{1} \phi_{t} \phi d x & =-\frac{1}{\beta_{1}} \int_{0}^{1} \phi_{x} \phi d x+\frac{K}{\beta_{1}} \int_{0}^{1} \phi_{x x} \phi d x+\frac{\left(\beta_{2}-\beta_{1}\right)}{\beta_{1}} \int_{0}^{1}\left(\mathcal{A} c_{2}\right) \phi d x \\
& =-\frac{1}{2 \beta_{1}} \int_{0}^{1}\left(\phi^{2}\right)_{x} d x-\frac{K}{\beta_{1}} \int_{0}^{1}\left(\phi_{x}\right)^{2} d x+\frac{\left(\beta_{2}-\beta_{1}\right)}{\beta_{1}} \int_{0}^{1}\left(\mathcal{A} c_{2}\right) \phi d x .
\end{aligned}
$$

Since $0<m<\beta_{i}(t)<\mathcal{M}, i=1,2$, and $\phi(0, t)=0$, it follows that

$$
\frac{1}{2} \frac{d}{d t}\left(\|\phi\|_{L^{2}(0,1)}^{2}\right) \leq \frac{2 \mathcal{M}}{m}\left\|\mathcal{A} c_{2}\right\|_{L^{2}(0,1)}\|\phi\|_{L^{2}(0,1)} .
$$

By results in [14] or [17], there exists a constant $C_{1}$ such that

$$
\left\|\mathcal{A} c_{2}\right\|_{L^{2}(0,1)} \leq \frac{C_{1} \beta_{2}(0)}{t}\left\|c_{0}\right\|_{L^{2}(0,1)}
$$

Hence

$$
\frac{d}{d t}\left(\|\phi\|_{L^{2}(0,1)}\right) \leq \frac{2 \mathcal{M} C_{1} \beta_{2}(0)}{m t}\left\|c_{0}\right\|_{L^{2}(0,1)} .
$$

Integrating this over $\left(t_{1}, t_{2}\right)$ yields

$$
\begin{aligned}
\|\phi\|_{L^{2}(0,1)}\left(t_{2}\right) & \leq\|\phi\|_{L^{2}(0,1)}\left(t_{1}\right)+\left(2 \mathcal{M} C_{1} \beta_{2}(0)\left\|c_{0}\right\|_{L^{2}(0,1)} / m\right) \ln \left|t_{2} / t_{1}\right| \\
& \leq\|\phi\|_{L^{2}(0,1)}\left(t_{1}\right)+\left(2 \mathcal{M} C_{1} \beta_{2}(0)\left\|c_{0}\right\|_{L^{2}(0,1)} / m\right) \ln |1+\varepsilon|,
\end{aligned}
$$

where $\varepsilon=\left(t_{2}-t_{1}\right) / t_{1}$. Since $t_{2}$ can be chosen to be arbitrarily close to $t_{1}$, and since $\ln (1+z)<z$ for all $z>0$, it follows that

$$
\|\phi\|_{L^{2}(0,1)}\left(t_{2}\right) \leq\|\phi\|_{L^{2}(0,1)}\left(t_{1}\right)+\left(2 \mathcal{M} C_{1} \beta_{2}(0)\left\|c_{0}\right\|_{L^{2}(0,1)} / m\right) \varepsilon
$$


for all $\varepsilon>0$. Therefore $\|\phi\|_{L^{2}(0,1)}\left(t_{2}\right) \leq\|\phi\|_{L^{2}(0,1)}\left(t_{1}\right)$ or

$$
\left\|\beta_{1} c_{1}-\beta_{2} c_{2}\right\|_{L^{2}(0,1)}\left(t_{2}\right) \leq\left\|\beta_{1} c_{1}-\beta_{2} c_{2}\right\|_{L^{2}(0,1)}\left(t_{1}\right) .
$$

Letting $t_{1}$ approach 0 , we have

$$
\left\|\beta_{1} c_{1}-\beta_{2} c_{2}\right\|_{L^{2}(0,1)}(t) \leq\left\|\beta_{1} c_{1}-\beta_{2} c_{2}\right\|_{L^{2}(0,1)}(0)=\left|\beta_{1}(0)-\beta_{2}(0)\right|\left\|c_{0}\right\|_{L^{2}(0,1)}=0
$$

for small $t$. This implies that $\beta_{1}(t) c_{1}(x, t)-\beta_{2}(t) c_{2}(x, t)=0$ almost everywhere on $[0,1]$. It follows from $c_{1}(1, t)=c_{2}(1, t)$ that $\beta_{1}(t)=\beta_{2}(t)$ for small $t$. Repeated application of this argument extends the result to $[0, T]$.

\subsection{Output least squares and Tikhonov regularization. We define}

$$
G(\beta) \equiv \gamma c_{\beta}
$$

with

$$
G: \mathcal{B} \rightarrow L^{2}(0, T)
$$

In the presence of perfect data $z$, we would solve the nonlinear ill-posed problem

$$
G\left(\beta^{0}\right)=z,
$$

where $c_{\beta}$ is the solution of the direct problem with $\beta=\beta^{0}$. To do this using Tikhonov regularization would involve approximating the solution by minimizing

$$
\min _{\beta \in \mathcal{B}}\|G(\beta)-z\|_{L^{2}(0, T)}^{2}+\alpha\|\beta-\hat{\beta}\|_{L^{2}(0, T)}^{2}
$$

where $\alpha>0$ is a small parameter and $\hat{\beta}$ is an a priori guess of the true solution $\beta^{0}$. In real applications, measurement errors mean that exact data is not available. Noisy data is assumed to have an error level $\delta$,

$$
\left\|z^{\delta}-z\right\|_{L^{2}(0, T)} \leq \delta
$$

We assume attainability of a true solution; i.e., if $z \in L^{2}(0, T)$, there exists $\beta^{0} \in \mathcal{B}$ such that

$$
G\left(\beta^{0}\right)=z
$$

We seek the minimizer $\beta_{\alpha}^{\delta} \in \mathcal{B}$ of

$$
J_{\alpha}(\beta)=\left\|G(\beta)-z^{\delta}\right\|_{L^{2}(0, T)}^{2}+\alpha\|\beta-\hat{\beta}\|_{L^{2}(0, T)}^{2}
$$

for appropriate choices of $\hat{\beta} \in \mathcal{B}$ and $\alpha$. We begin by establishing the weak-closedness of the map $\beta \rightarrow \gamma c_{\beta}$. This will lead to the existence of a minimizer $\beta_{\alpha}^{\delta}$. Continuous dependence on the data $z^{\delta}$ for fixed $\alpha$, and the convergence of $\beta_{\alpha}^{\delta}$ toward the true parameter $\beta^{0}$ as the noise level $\delta$ and the regularization parameter $\alpha$ go to zero, also follow.

TheOREM 3.3. If $\beta_{n} \rightarrow \beta_{*} \in \mathcal{B}$ in $H^{1}(0, T)$, then $c_{\beta_{n}} \rightarrow c_{\beta_{*}}$ in $W$ and $\gamma c_{\beta^{n}} \rightarrow$ $\gamma c_{\beta}^{*}$ in $L^{2}(0, T)$. 
Proof. Since we have existence of a unique solution to (3.1) from Theorem 3.1, we define $c_{n}=c\left(\beta_{n}\right)$. We make a change of variables, $w=e^{-\lambda t} c$, where $\lambda$ is to be chosen. The state equation and initial and boundary conditions become

$$
\begin{array}{r}
(\beta w)_{t}+\lambda \beta w-K w_{x x}+w_{x}=0, \\
w(0, t)=0, w_{x}(1, t)=0, \quad t \in[0, T], \\
w(x, 0)=c_{0}(x) .
\end{array}
$$

Let $\langle\cdot, \cdot\rangle$ denote the duality between $\left(H^{1}(0,1)\right)^{*}$ and $H^{1}(0,1)$. We use the weak definition of the transformed equation and integrate in time to obtain

$$
0=\int_{0}^{t}\left\langle\left(\beta_{n} w_{n}\right)_{t}, \beta_{n} w_{n}\right\rangle d t+\int_{0}^{t} \int_{0}^{1}\left[\lambda\left(\beta_{n} w_{n}\right)^{2}+K \beta_{n}\left(w_{n}\right)_{x}^{2}+\beta_{n}\left(w_{n}\right)_{x} w_{n}\right] d x d t .
$$

Upon simplification, use of $0<m<\beta_{n}(t)<\mathcal{M}$, and Cauchy's inequality, we have

$$
\begin{aligned}
& \frac{m^{2}}{2} \int_{0}^{1}\left[w_{n}(x, t)\right]^{2} d x+\lambda m^{2} \int_{0}^{t} \int_{0}^{1}\left(w_{n}\right)^{2} d x d t+K m \int_{0}^{t} \int_{0}^{1}\left(w_{n}\right)_{x}^{2} d x d t \\
\leq & \frac{\mathcal{M}^{2}}{2} \int_{0}^{1}\left[c_{0}(x)\right]^{2} d x+\frac{\mathcal{M}^{2}}{2 K m} \int_{0}^{t} \int_{0}^{1}\left(w_{n}\right)^{2} d x d t+\frac{K m}{2} \int_{0}^{t} \int_{0}^{1}\left(w_{n}\right)_{x}^{2} d x d t .
\end{aligned}
$$

After dividing by $m^{2}$, collecting terms, and choosing $\lambda>\frac{\left(2 K m^{3}+1\right) \mathcal{M}^{2}}{2 K m^{3}}$, we obtain

$$
\begin{aligned}
& \int_{0}^{1}\left[w_{n}(x, t)\right]^{2} d x d t+\frac{K}{m} \int_{0}^{t} \int_{0}^{1}\left(w_{n}\right)_{x}^{2} d x d t+2 \mathcal{M}^{2} \int_{0}^{t} \int_{0}^{1}\left(w_{n}\right)^{2} d x d t \\
& \quad \leq \frac{\mathcal{M}^{2}}{m^{2}} \int_{0}^{1}\left[c_{0}(x)\right]^{2} d x .
\end{aligned}
$$

We can conclude that $\left\|w_{n}\right\|_{L^{2}\left((0, T), H^{1}(0,1)\right)}$, and hence $\left\|c_{n}\right\|$ is uniformly bounded independent of $n$. Using this bound and the state equation, we also have uniform bounds on $\left\|\left(\beta_{n} c_{n}\right)_{t}\right\|$. We can extract a subsequence such that

$$
\begin{aligned}
c_{n} & \rightarrow c_{*} \quad \text { in } \quad L^{2}\left((0, T) ; H^{1}(0,1)\right), \\
\left(c_{n}\right)_{t} & \rightarrow\left(c_{*}\right)_{t} \quad \text { in } \quad L^{2}\left((0, T) ;\left(H^{1}(0,1)\right)^{*}\right), \\
\beta_{n} & \rightarrow \beta_{*} \quad \text { in } \quad L^{2}(0, T),
\end{aligned}
$$

where $c_{*}$ and $\beta_{*}$ are the relevant weak limits. In order to show that $c^{*}=c\left(\beta_{*}\right)$, we must establish that $c_{*}$ is the state solution associated with $\beta_{*}$. We consider the weak form of the partial differential equation satisfied by $c_{n}$,

$$
\int_{0}^{T}\left\langle\left(\beta^{n} c^{n}\right)_{t}, \phi\right\rangle d t+K \int_{0}^{T} \int_{0}^{1} c_{x}^{n} \phi_{x} d x d t+\int_{0}^{T} \int_{0}^{1} c_{x}^{n} \phi d x d t=0,
$$

where $\phi \in L^{2}\left((0, T) ; H^{1}(0,1)\right)$.

Since $\beta_{n} \rightarrow \beta_{*}$ in $L^{2}(0, T)$ we know that $\beta_{n} \rightarrow \beta_{*}$ in $H^{1}(0, T)$. Therefore $\left(\beta_{n}\right)^{\prime} \rightarrow$ $\left(\beta_{*}\right)^{\prime}$ in $L^{2}(0, T)$, where ${ }^{\prime}$ is used to indicate the derivative here because $\beta$ is a function of one variable, $t$. We examine the first term in the weak definition of state solution,

$$
\begin{aligned}
& \int_{0}^{T} \int_{0}^{1}\left[\left(\beta_{n} c_{n}\right)_{t}-\left(\beta_{*} c_{*}\right)_{t}\right] \phi d x d t \\
= & \int_{0}^{T} \int_{0}^{1} \begin{array}{r}
{\left[\left(\beta_{n}\right)^{\prime}\left[c_{n}-c_{*}\right] \phi+\left(\beta_{n}-\beta_{*}\right)^{\prime} c_{*} \phi\right.} \\
\left.\quad+\beta_{n}\left(\left(c_{n}\right)_{t}-\left(c_{*}\right)_{t}\right) \phi+\left(\beta_{n}-\beta_{*}\right)\left(c_{*}\right)_{t} \phi\right] d x d t .
\end{array}
\end{aligned}
$$


We note that, from a comparison result in [20], we have that $c_{n} \rightarrow c_{*}$ in $L^{2}((0,1) \times$ $(0, T))$. By $\left(\beta_{n}\right)^{\prime} \rightarrow\left(\beta_{*}\right)^{\prime}$ in $L^{2}(0, T)$ and $c_{n} \rightarrow c_{*}$ in $L^{2}((0,1) \times(0, T))$, the first term of (3.9) converges to zero as $n \rightarrow \infty$. The second term converges to zero since $\left(\beta_{n}\right)^{\prime} \rightarrow\left(\beta_{*}\right)^{\prime}$ in $L^{2}(0, T)$. The third and fourth terms converge to zero because of the strong convergence of the $\beta_{n}$ sequence. As we pass to the limit in the weak definition of the solution, we obtain that $c_{*}=c\left(\beta_{*}\right)$.

Existence of a minimizer $\beta_{\alpha}^{\delta}$ now follows from the lower semicontinuity of the $L^{2}(0, T)$ norm. Continuous dependence on the data $z^{\delta}$ for fixed $\alpha$ and the convergence of $\beta_{\alpha}^{\delta}$ toward the true parameter $\beta^{0}$ follow from standard results [19].

Corollary 3.4. For any data $z^{\delta} \in L^{2}(0, T)$, a minimizer $\beta_{\alpha}^{\delta}$ of $(3.5)$ exists.

Corollary 3.5. For fixed $\alpha$, the minimizers depend continuously on the data $z^{\delta}$. If $\alpha(\delta)$ satisfies

$$
\alpha(\delta) \rightarrow 0, \quad \delta^{2} / \alpha(\delta) \rightarrow 0 \quad \text { as } \delta \rightarrow 0,
$$

then

$$
\lim _{\delta \rightarrow 0}\left\|\beta_{\alpha}^{\delta}-\beta^{0}\right\|_{L^{2}(0, T)}=0 .
$$

3.3. Convergence rates. Although we have established convergence of the minimizer $\beta_{\alpha}^{\delta}$ to the true parameter $\beta^{0}$, the rate of convergence may be arbitrarily slow. We apply the theory of Engl, Hanke, and Neubauer [7] and Engl, Kunisch, and Neubauer [8] to determine a source condition that will guarantee a certain rate of convergence. Recall that we seek to solve the nonlinear problem $(3.3), G(\beta)=z$, where $G(\beta) \equiv \gamma c_{\beta}$. The true solution is $\beta^{0}$, and $\hat{\beta}$ is an a priori guess. Let $L(\beta)$ be the differential operator

$$
L(\beta) u \equiv(\beta u)_{t}+u_{x}-K u_{x x}
$$

on the domain $D(L)=\left\{u \in W \mid u(0, t)=u_{x}(1, t)=0\right\}$. The soil problem (3.1) satisfies

$$
L(\beta) c=0, \quad c(x, 0)=c_{0}(x) .
$$

We establish next an estimate of the rate of convergence of our algorithm. Even when our regularization parameter $\alpha$ is comparable to our noise level $\delta$, convergence requires assumptions involving $c(1, t)$ and $\beta^{0}-\hat{\beta}$.

THEOREM 3.6. Let $B y \equiv-y^{\prime \prime}+y$ with

$$
B: D(B)=\left\{y \in H^{2}(0, T) \mid y^{\prime}(0)=y^{\prime}(T)=0\right\} \rightarrow L^{2}(0, T) .
$$

If

$$
\frac{B\left(\beta^{0}(t)-\hat{\beta}(t)\right)}{c(1, t)} \in H^{-1}(0, T)
$$

and if

$$
\left\|\int_{t}^{T} \frac{B\left(\beta^{0}(t)-\hat{\beta}\left(t^{\prime}\right)\right)}{c\left(1, t^{\prime}\right)} d t^{\prime}\right\|_{L^{2}(0, T)} \quad \text { is sufficiently small, }
$$


then for the choice $\alpha \sim \delta$ we obtain

$$
\left\|\beta_{\alpha}^{\delta}-\beta^{0}\right\|_{H^{1}(0, T)}=O(\sqrt{\delta}) \quad \text { and } \quad\left\|G\left(\beta_{\alpha}^{\delta}\right)-z^{\delta}\right\|_{L^{2}(0, T)}=O(\delta) .
$$

Proof. We define

$$
F(\beta) \equiv c(x, t) .
$$

If $\Psi_{h}(x, t)=F^{\prime}(\beta) h(t)$ is the first Fréchet derivative in the direction $h$, then $\Psi_{h} \in$ $D(L)$ and

$$
L(\beta) \Psi_{h}=-(h c)_{t}, \quad \Psi_{h}(x, 0)=0 .
$$

Similarly, if $\Phi_{h}(x, t)=F^{\prime \prime}(\beta)(h(t), h(t))$ is the second Fréchet derivative in the direction $h$, then $\Phi_{h} \in D(L)$ and

$$
L(\beta) \Phi_{h}=-2\left(h \Psi_{h}\right)_{t}, \quad \Phi_{h}(x, 0)=0 .
$$

By continuity of the trace operator, we have

$$
G^{\prime}(\beta) h(t)=\Psi_{h}(1, t)=\gamma \Psi_{h}, \quad G^{\prime \prime}(\beta)(h(t), h(t))=\Phi_{h}(1, t)=\gamma \Phi_{h} .
$$

Thus, $G$ is twice Fréchet differentiable.

Define $p \in W$ to be the solution of

$$
L(\beta)^{*} p=h, \quad p(x, T)=0,
$$

where the adjoint $L^{*}$ is taken with respect to $L^{2}(0, T)$. Notice that

$$
\begin{aligned}
\left\langle g, G^{\prime}(\beta)^{*} h\right\rangle_{H^{1}(0, T)} & =\left\langle G^{\prime}(\beta) g, h\right\rangle_{L^{2}(0, T)} \\
& =\left\langle\gamma \Psi_{g}, h\right\rangle_{L^{2}(0, T)}=\gamma\left\langle\Psi_{g}, h\right\rangle_{L^{2}(0, T)} \\
& =\gamma\left\langle-L^{-1}(g c)_{t}, h\right\rangle_{L^{2}(0, T)}=-\gamma\left\langle(g c)_{t},\left(L^{-1}\right)^{*} h\right\rangle_{L^{2}(0, T)} \\
& =-\gamma\left\langle(g c)_{t},\left(L^{*}\right)^{-1} h\right\rangle_{L^{2}(0, T)}=-\gamma\left\langle(g c)_{t}, p\right\rangle_{L^{2}(0, T)} \\
& =\gamma\left\langle g, c p_{t}\right\rangle_{L^{2}(0, T)},
\end{aligned}
$$

provided that $g(0)=0$. Since

$$
\langle g, v\rangle_{L^{2}(0, T)}=\left\langle\left(B^{-1}\right)^{*} g, v\right\rangle_{H^{1}(0, T)}
$$

for every $v \in H^{1}(0, T)$, it follows that

$$
\gamma\left\langle g,\left(c p_{t}\right)\right\rangle_{L^{2}(0, T)}=\gamma\left\langle\left(B^{-1}\right)^{*} g, c p_{t}\right\rangle_{H^{1}(0, T)}=\gamma\left\langle g, B^{-1}\left(c p_{t}\right)\right\rangle_{H^{1}(0, T)} .
$$

This means that

$$
G^{\prime}(\beta)^{*} h=\gamma B^{-1}\left(c p_{t}\right)
$$

with $L(\beta)^{*} p=h, p(x, T)=0$. Since (3.10) holds, there exists $w \in L^{2}(0, T)$ such that

$$
\beta^{0}-\hat{\beta}=G^{\prime}\left(\beta_{0}\right)^{*} w
$$

or $B\left(\beta^{0}-\hat{\beta}\right)=c(1, t) p_{t}(1, t)$ with

$$
L\left(\beta^{0}\right) c=0, c(x, 0)=c_{0}(x), \quad L\left(\beta^{0}\right)^{*} p=w(t), p(x, T)=0 .
$$


Let $h:=\beta_{\alpha}^{\delta}-\beta^{0}$ and $\beta^{s}:=\beta^{0}+s h$. Then

$$
\begin{aligned}
\mid 2\langle w & \left., \int_{0}^{1} G^{\prime \prime}\left(\beta^{s}\right)(h, h)(1-s) d s\right\rangle_{L^{2}(0, T)} \mid \\
& =\left|2 \int_{0}^{T} w(t) \int_{0}^{1} G^{\prime \prime}\left(\beta^{s}(t)\right)(h(t), h(t))(1-s) d s d t\right| \\
& \leq \sup _{0 \leq s \leq 1}\left|\left\langle w, G^{\prime \prime}\left(\beta^{s}\right)(h, h)\right\rangle_{L^{2}(0, T)}\right| \\
& =\sup _{0 \leq s \leq 1}\left|\left\langle\gamma L\left(\beta^{0}\right)^{*} p,, G^{\prime \prime}\left(\beta^{s}\right)(h, h)\right\rangle_{L^{2}(0, T)}\right| \\
& =\|\gamma p\|_{L^{2}(0, T)}\left\|\gamma L\left(\beta^{0}\right) \Psi_{h}\right\|_{L^{2}(0, T)} .
\end{aligned}
$$

As in [8], it can be shown that there exist constants $C_{1}$ and $C_{2}$ such that

$$
\left\|\Phi_{h}\right\|_{W} \leq C_{1}\|h\|_{L^{2}(0, T)}\left\|\Psi_{h}\right\|_{W}, \quad\left\|\Psi_{h}\right\|_{W} \leq C_{2}\|h\|_{L^{2}(0, T)}\|c\|_{W} .
$$

By the trace theorem $\left[9\right.$, p. 258] and boundedness of the operator $L\left(\beta^{0}\right)$, there exists $C_{3}$ such that

$$
\left\|\gamma L\left(\beta^{0}\right) \Psi_{h}\right\|_{L^{2}(0, T)} \leq C_{3}\|h\|_{H^{1}(0, T)}^{2}\|c\|_{W} .
$$

As the quantity appearing in (3.11) is $\|\gamma p\|_{L^{2}(0, T)}$, we deduce that

$$
2\left\langle w, \int_{0}^{1} G^{\prime \prime}\left(\beta^{s}\right)(h, h)(1-s) d s\right\rangle_{L^{2}(0, T)} \leq \rho\|h\|_{H^{1}}^{2}
$$

with $\rho<1$. Since $G$ is twice Fréchet differentiable and both (3.12) and (3.13) hold, application of the theory of Engl, Kunisch, and Neubauer [8, Theorem 2.4] yields the desired convergence result.

Discussion of source condition. The condition (3.10) requires that the difference between the a priori guess $\hat{\beta}$ and the true solution $\beta^{0}$ must be in $D(B) \subset H^{2}(0, T)$. In practical applications, this regularity assumption is very restrictive.

It was established in Oppenheimer [14] for Hölder continuous $\beta$ that there exist constants $C \geq 0$ and $\theta>0$ such that

$$
\|c(\cdot, t)\|_{L^{2}(0,1)} \leq C \frac{\beta(0)}{\beta(t)} e^{-\theta t}\left\|c_{0}\right\|_{L^{2}(0,1)} .
$$

Since $\beta \in \mathcal{B}$ is bounded, there exist constants $C \geq 0$ and $\theta>0$ such that

$$
\|c(\cdot, t)\|_{L^{2}(0,1)} \leq C e^{-\theta t}\left\|c_{0}\right\|_{L^{2}(0,1)} .
$$

The requirement (3.11) means that the difference between our a priori guess $\hat{\beta}$ and the true parameter $\beta^{0}$ must be small and very smooth when our measurement $c(1, t)$ is small. This is both a local and global restriction. Since $c(\cdot, t)$ decays exponentially, this is possible only for sufficiently small $T$. 


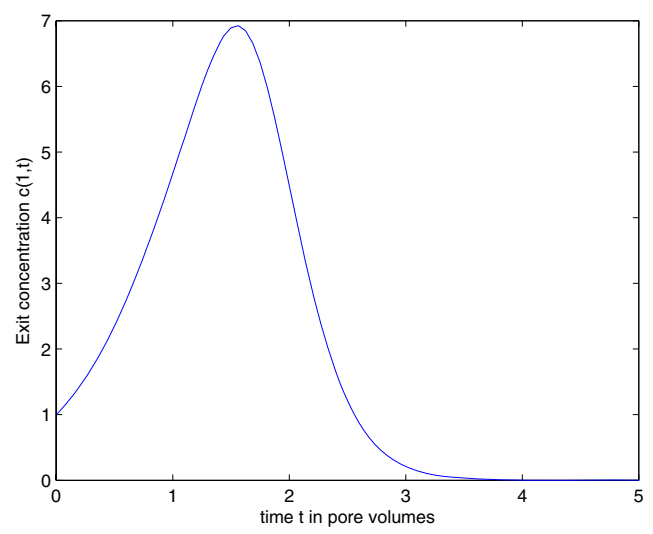

FIG. 4.1. Exit concentration.

4. Numerical results. In order to demonstrate the effectiveness of Tikhonov regularization for this application, we consider two examples. Recall that the solution of our forward problem (3.1) decays over time, i.e., $c(x, t) \rightarrow 0$ as $t \rightarrow \infty$. As a result, we do not expect to be able to use all of the available data or to recover $\beta(t)$ over the whole time interval.

All computations were carried out in MATLAB. The Tikhonov functional

$$
J_{\alpha}(\beta)=\int_{0}^{T}\left(c(1, t)-z^{\delta}\right)^{2} d t+\alpha \int_{0}^{T}(\beta(t)-\hat{\beta}(t))^{2} d t
$$

was minimized using a Gauss-Newton method. Here $z^{\delta}$ and $\hat{\beta}$ represent noisy data and an a priori guess of the parameter. During the computation of $J_{\alpha}(\beta)$, exit concentrations $c(1, t)$ associated with a particular $\beta$ were computed using an implicit finite-difference algorithm. The integrals were computed using a trapezoidal rule. Exit concentration data was generated using a method of lines algorithm with high accuracy. The a priori guess was chosen to be $\hat{\beta}=\beta(0)$, which was estimated using the approximation $\beta(0) \approx\left(\int_{0}^{t_{N}} c(1, t) d t\right) / c_{0}$. Strategies for the discussion of regularization parameters are discussed in [21]. For the purposes of this discussion, we will choose the regularization parameter to be $\alpha=0$ in the absence of noise.

Example 1. Let $c_{0}(x)=1$ and $K=0.07$. Consider the sorption coefficient

$$
\beta(t)=1+10 e^{-2 t}
$$

The exit concentration $c(1, t)$ associated with this $\beta$ is shown in Figure 4.1. Since the data decays over time, we restrict the recovery of $\beta$ to the time interval $[0,2.5]$. An initial guess of $\beta=1$ was used. The parameter $\beta$ and its recovery $\beta_{\text {nonoise }}$ from noiseless data are shown in Figure 4.2. Notice that quality of the recovery degrades after $t=2$. This is due to the fact that the exit concentrations become very small and begin to amplify numerical error in the algorithm.

Example 2. Let $c_{0}(x)=1$ and $K=0.07$. Consider the sorption coefficient

$$
\beta(t)=2+\cos (10 t) .
$$

The exit concentration $c(1, t)$ associated with this $\beta$ is shown in Figure 4.3. Once again the data decays over time, and we restrict the recovery of $\beta$ to the time interval $[0,2]$. 


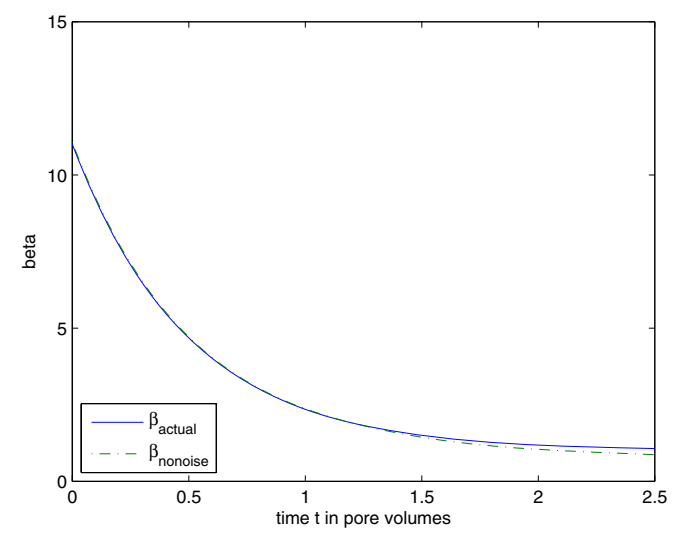

FIG. 4.2. $\beta_{\text {nonoise }}$ recovered from exact data.

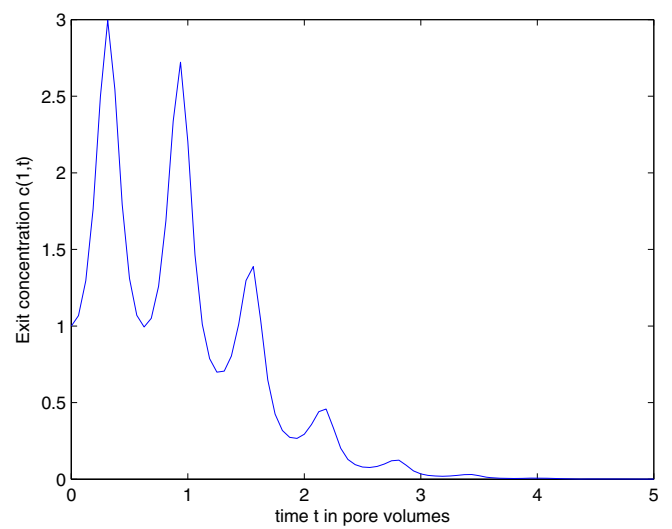

Fig. 4.3. Exit concentration.

An initial guess of $\beta=2$ was used. The parameter $\beta$ and its recovery $\beta_{\text {nonoise }}$ from noiseless data are shown in Figure 4.4. Notice that quality of the recovery degrades after $t=1.25$.

Example 3: Noisy data. Since data for this problem is measured experimentally, it will contain a certain amount of noise. The nature of these experiments suggests that the noise level may be as much as $20 \%$. Noise is introduced into the data from Example 1 via a normally distributed random number generator. Figure 4.5 shows the noisy exit concentration data.

In engineering applications, it is not practical to expect to have sufficient information about the unknown parameter $\beta^{0}$ in order to choose an a priori guess $\hat{\beta}$ so that $\beta^{0}-\hat{\beta} \in D(B) \subset H^{2}(0, T)$. Hence Theorem 3.6 does not apply. Instead, a regularization parameter of $\alpha=10^{-3}$ was chosen heuristically by an L-curve method, [21]. Figure 4.6 shows the recovery of $\beta_{\text {noise }}$ with and without regularization. Clearly $\alpha=10^{-3}$ produces better results than $\alpha=0$, although the recovery is not as good as the noiseless case in Example 1. 


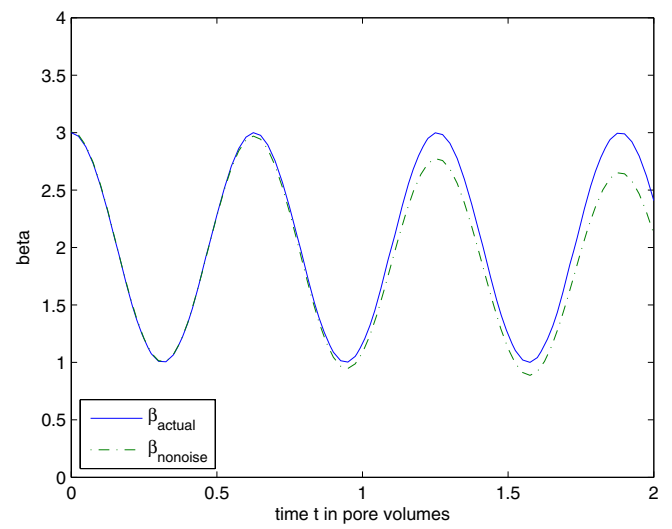

FIG. 4.4. $\beta_{\text {nonoise }}$ recovered from exact data.

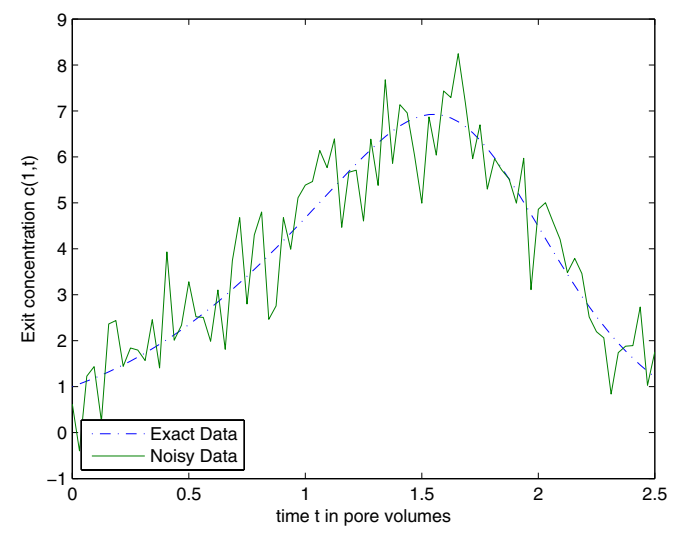

FIG. 4.5. Exit concentration data with $20 \%$ noise.
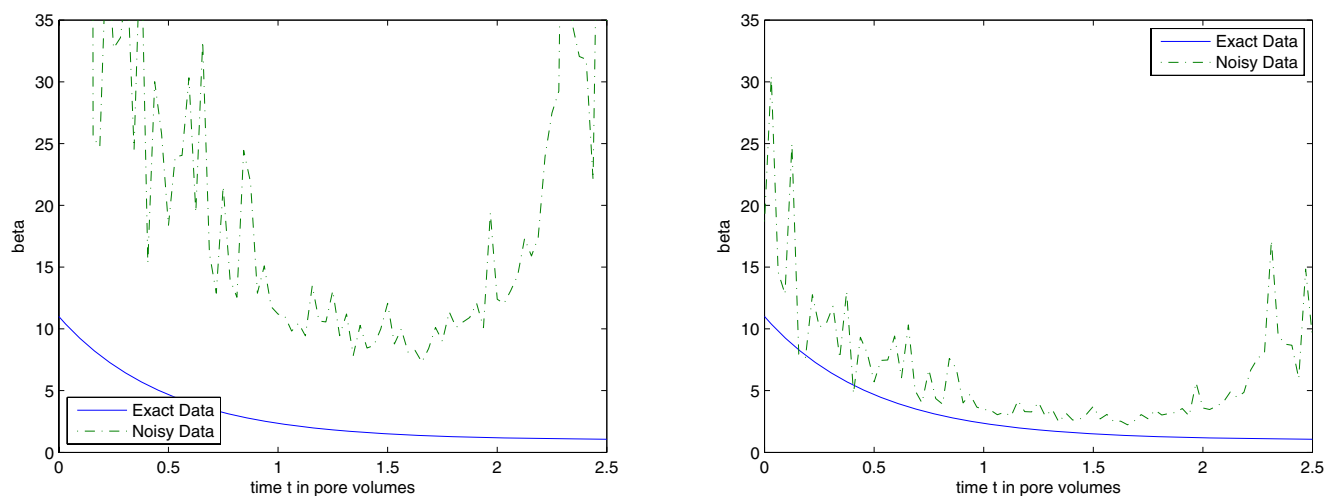

FIG. 4.6. $\beta_{\text {noise }}$ recovered from noisy data without regularization and with a regularization parameter of $\alpha=10^{-3}$. 
5. Conclusions. In this paper, we have identified the sorption partitioning coefficient as a function of time from limited boundary data. A numerical approach for approximating this time dependent parameter in a parabolic partial differential equation has been analyzed. This work has brought insight into how a partitioning coefficient varies with different physical factors such as temperature fluctuations and contaminant introduction in the soil column. In the column studies, the boundary data is represented by the measurements of contaminant concentrations as the solution exits the soil column. The identifiability of the soil sorption parameter, $\beta$, is determined from these noisy exit concentration measurements. In order to establish the identifiability of the parameter $\beta$, we proved the injectivity of the parameter to the output map. We then discussed an output least squares formulation of the problem with Tikhonov regularization. Using this format, we found a minimizer to our approximate problem and were able to prove that this minimizer converges to the true parameter as the noise level and the regularization parameter approach zero. Although we proved convergence, the rate of convergence may be arbitrarily slow. Therefore, we established a source condition that guarantees a given rate of convergence. However, there is a trade-off here. The condition requires that the difference between our a priori guess and the true parameter must be small and relatively smooth when the boundary measurements are small. We found that this in possible only over a small time interval because the contaminant concentration decays over time. Within the numerical examples, this is seen after $t=2$ in Example 1 and after $t=1.6$ in Example 2. However, with the noisy data, the quality of the identification significantly improves with the inclusion of a regularization parameter. Consequently, the implementation of Tikhonov regularization provides a more tractable result.

\section{REFERENCES}

[1] D. D. Adrian, S. Ozkan, And A. N. Alshawabkeh, Tracer transport in a soil column for sine wave loading, in Physical and Chemical Processes of Water and Solute Transport/Retention in Soil, H. M. Selim and D. L. Sparks, eds., Special Publication 56, Soil Science Society of America, Madison, WI, 2001, pp. 169-188.

[2] J. R. CANnon, Determination of an unknown coefficient in a parabolic differential equation, Duke Math J., 30 (1963), pp. 313-324.

[3] J. R. Cannon, P. DuChateau, and K. Steube, Unknown ingredient inverse problems and trace-type functional differential equations, in Inverse Problems in Partial Differential Equations, D. Colton, R. Ewing, and W. Rundell, eds., SIAM, Philadelphia, 1990, pp. $187-202$.

[4] J. R. CANnon, Y. Lin, AND S. Xu, Numerical procedures for the determination of an unknown coefficient in semi-linear parabolic differential equations, Inverse Problems, 10 (1994), pp. $227-243$.

[5] J. R. Cannon and W. Rundell, Recovering a time-dependent coefficient in a parabolic differential equation, J. Math. Anal. Appl., 160 (1991), pp. 572-582.

[6] P. A. Domenico and F. W. Schwartz, Physical and Chemical Hydrogeology, 2nd ed., John Wiley \& Sons, New York, 1998.

[7] H. W. Engl, M. Hanke, And A. Neubauer, Regularization of Inverse Problems, Kluwer Academic Publishers, Norwell, MA, 2000.

[8] H. W. Engl, K. Kunisch, And A. Neubauer, Convergence rates for Tikhonov regularisation of non-linear ill-posed problems, Inverse Problems, 5 (1989), pp. 523-540.

[9] L. C. Evans, Partial Differential Equations, American Mathematical Society, Providence, RI, 1998.

[10] A. G. Fatullayev, Numerical procedure for the simultaneous determination of unknown coefficients in a parabolic equation, Appl. Math. Comput., 164 (2005), pp. 697-705.

[11] V. Isakov, Inverse Problems for Partial Differential Equations, Springer, New York, 1998.

[12] F. J. Leij And J. H. Dane, A Review of Physical and Chemical Processes Pertaining to Solute Transport, Agronomy and Soils Departmental Series 132, Alabama Agricultural Experiment Station, Auburn University, Auburn, AL, 1989. 
[13] T. E. Myers, Metals Release from Freshwater and Estuarine Sediments in Thin-Disk Leaching Columns, Ph.D. thesis, Civil and Environmental Engineering Department, Louisiana State University and Agricultural and Mechanical College, Baton Rouge, LA, 1999.

[14] S. F. Oppenheimer, Parameter Identification for an Advection-Dispersion Equation with Salinity Controlled Partitioning, Technical report DACW 39-92-M-6776, U.S. Army Engineers, Waterways Experiment Station, Vicksburg, MS, 1992.

[15] S. F. OpPenheimer, The sorption of mixtures under linear equilibrium partitioning and chemical transformation, Math. Methods Appl. Sci., 18 (1995), pp. 803-823.

[16] S. F. Oppenheimer, W. L. Kingery, and F. X. Han, Phase plane analysis and dynamical systems approaches to the study of metal sorption in soils, in Heavy Metals Release in Soils, H. M. Selim and D. L. Sparks, eds., Lewis Publishers, New York, 2001, pp. 109-130.

[17] A. PAZY, Semigroups of Linear Operators and Applications to Partial Differential Equations, Springer, New York, 1983.

[18] M. S. Pilant AND W. Rundell, Undetermined coefficient problems for quasilinear parabolic equations, in Inverse Problems in Partial Differential Equations, D. Colton, R. Ewing, and W. Rundell, eds., SIAM, Philadelphia, 1990, pp. 165-185.

[19] T. I. Seidman And C. R. Vogel, Well-posedness and convergence of some regularization methods for non-linear ill-posed problems, Inverse Problems, 5 (1989), pp. 227-238.

[20] J. Simon, Compact sets in the space $L^{p}(0, T ; B)$, Ann. Mat. Pura Appl. (4), 146 (1987), pp. 65-96.

[21] C. R. Vogel, Computational Methods for Inverse Problems, Frontiers in Appl. Math. 23, SIAM, Philadelphia, 2002. 